\title{
Dynamic measurements of the elastic constants of glass wool
}

\author{
Tarnow, Viggo
}

Published in:

Acoustical Society of America. Journal

Link to article, DOI:

$10.1121 / 1.2118267$

Publication date:

2005

Document Version

Publisher's PDF, also known as Version of record

Link back to DTU Orbit

Citation (APA):

Tarnow, V. (2005). Dynamic measurements of the elastic constants of glass wool. Acoustical Society of America. Journal, 118(6), 3672-3678. https://doi.org/10.1121/1.2118267

\section{General rights}

Copyright and moral rights for the publications made accessible in the public portal are retained by the authors and/or other copyright owners and it is a condition of accessing publications that users recognise and abide by the legal requirements associated with these rights.

- Users may download and print one copy of any publication from the public portal for the purpose of private study or research.

- You may not further distribute the material or use it for any profit-making activity or commercial gain

- You may freely distribute the URL identifying the publication in the public portal

If you believe that this document breaches copyright please contact us providing details, and we will remove access to the work immediately and investigate your claim 


\title{
Dynamic measurements of the elastic constants of glass wool
}

\author{
Viggo Tarnow ${ }^{a)}$ \\ Department of Mechanical Engineering, Technical University of Denmark, Bygning 404, \\ DK 2800 Lyngby, Denmark
}

(Received 29 November 2004; revised 16 September 2005; accepted 16 September 2005)

\begin{abstract}
The sound wave in the air between the fibers of glass wool exerts an oscillatory viscous drag on the fibers and excites a mechanical wave in the fiber skeleton. Accurate calculations of sound attenuation in glass wool must take the mechanical wave in the fiber skeleton into account, and this requires knowledge of the dynamic elastic constants of the fiber skeleton. The mechanical properties of glass wool are highly anisotropic. Previously only one of the elastic constants has been measured dynamically, but here all the elastic constants are reported. The measurement method is well known. But a new mechanical design, which reduces mechanical resonance, is described. The measurements were carried out in atmospheric air at normal pressure, and this causes an oscillatory airflow in the sample. To obtain the elastic constants, the influence of the airflow was subtracted from the data by a new formula. The elastic constants were measured in the frequency range $20-160 \mathrm{~Hz}$ for glass wool of mass density $30 \mathrm{~kg} / \mathrm{m}^{3}$. The elastic constant $C_{11}$ depended on the frequency; at $20 \mathrm{~Hz}$ it was $1.5+0.01 i \mathrm{MPa}$, and at $160 \mathrm{~Hz}$ it was $2.6+0.06 i \mathrm{MPa}$. The constant $C_{33}=12+0.6 i \mathrm{kPa}$ did not depend on frequency. The shear constant $C_{44}=40+2 i \mathrm{kPa}$ was constant. The two constants $C_{12}, C_{13}$ were zero. (C) 2005 Acoustical Society of America. [DOI: 10.1121/1.2118267]
\end{abstract}

PACS number(s): 43.55.Ev, 43.20.Jr, 43.58.Dj [AJZ]

Pages: $3672-3678$

\section{INTRODUCTION}

Glass wool is placed inside the fuselage of airplanes, where it reduces the noise transmissions from the outside to the inside of the cabin. Glass wool is also placed inside walls in buildings, where it reduces the transmission of noise through the walls.

Experiments in the last five years have shown that sound waves, in the air between fibers, drag the skeleton of fiber materials, and this causes movements of the fibers, which decreases the attenuation of sound waves. ${ }^{1-4}$ Early measurements of sound attenuation in tubes did not show this, because samples were mounted in a way that prevented their movement. Recently ${ }^{2,3}$ measurements have been carried out with thin samples fixed to the cylindrical surface of the tube. In this case, the skeleton of the material in the center of the tube can move.

The airborne sound wave is coupled to the fiber skeleton borne wave by an oscillatory viscous drag of air on the fibers. To take this into account by the Biot theory, ${ }^{5}$ the dynamic elastic constants of the glass wool skeleton must be known.

Pritz $^{6}$ analyzed a measurement method where the glass wool sample is part of a spring as shown in Fig. 1. The shaker gave the lower part of the sample a displacement that was a sinusoidal function of time. Accelerometers measured the acceleration of the shaker table and the top plate. The amplitude and phase of the output from the two accelerometers were measured. From the complex ratio between the two accelerations, the length of the sample, the cross-section area, the mass of the sample, and the mass of the upper aluminum plate. Pritz calculated the complex Young modu-

${ }^{a)}$ Electronic mail: vt@mek.dtu.dk lus, and he made a detailed study of the measurement errors. Longitudinal wave movement was taken into account. It was assumed that the shaker and the upper aluminum plate moved translationally. In another paper ${ }^{7}$ Pritz reported measurement, with the same setup, of the complex Young's modulus of glass wool of density $71 \mathrm{~kg} / \mathrm{m}^{3}$ for frequency 100-3 $000 \mathrm{~Hz}$. The measurements were carried out in air and vacuum.

Rice and Göransson ${ }^{8}$ used a similar setup to measured a dynamic complex Young's modulus of glass wool with density 15 and $11 \mathrm{~kg} / \mathrm{m}^{3}$ for frequencies about 15 and $60 \mathrm{~Hz}$. Measurement were carried out in air and vacuum. Ingard ${ }^{9}$ used a similar setup to measure the complex Young's modulus of glass wool in vacuum.

To determine the complex Young's modulus of glass wool, Wilson and Cummings ${ }^{10}$ used a setup with a force transducer that measured the force in the sample. The frequency range was $40-200 \mathrm{~Hz}$. Figure 2 shows the principle. They first measured the complex mechanical impedance of one sample and then of a second sample with twice the thickness. From the two mechanical impedances and the length of the sample they calculated the complex wave number. The complex characteristic impedance was calculated from the measured impedances and the cross-sectional area. From frequency and the characteristic impedance, they calculated the effective mass density and Young's modulus. They assumed that their samples were homogeneous, and it was further assumed that there was no lateral movement of the samplethe Poisson's ratio was assumed to be zero. Langlois, Panneton, and Atalla ${ }^{11}$ used an experimental arrangement similar to Fig. 2 to measure the elastic properties of isotropic plastic foam. They developed a new way to find Young's modulus and Poisson's ratio from measurements on samples of different thickness. 


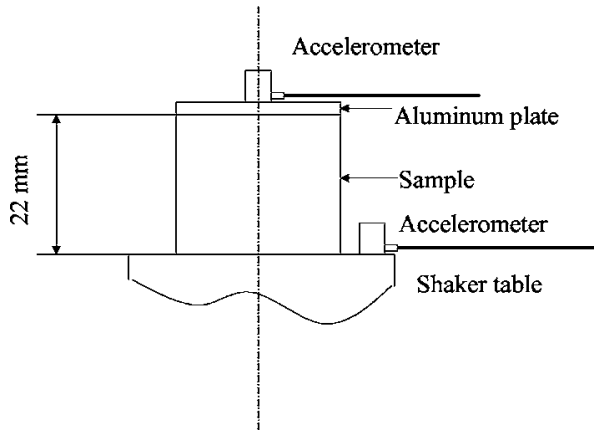

FIG. 1. Measurement principle with two moving surfaces, the sample is glued to the shaker table and the aluminum plate. The acceleration of the shaker table and the aluminum plate are measured.

The setup in Fig. 1 is attractive because it is simple. It was used in the beginning of the present study. The acceleration amplitude of the top plate and the shaker were recorded as a function of frequency. The acceleration amplitude of the top plate divided by the one of the shaker was plotted as a function of frequency. However, in many experiments the plot showed three resonance peaks between 200 and $300 \mathrm{~Hz}$ instead of only one as expected when the movement of the aluminum plate is a translation. The reason is that the top plate rocked, because the samples were not homogeneous. Pritz ${ }^{6}$ assumed a translation; Cremer, Heckl, and Ungar ${ }^{12}$ have observed excitation of rocking motions in similar experiments. Three modes of the top plate were excited. One mode is mainly a translation in the direction of dot-and-dash line in Fig. 1, the other modes are mainly rotations of the top plate. The movements of the top plate were detected by small accelerometers, which showed the accelerations of the three modes. Calculation of elastic constants from the data was unreliable, because three modes were excited.

The rotation of the upper plate was prevented in the setup in Fig. 2, and measurements reported in this paper were carried out with a similar setup. Measurements should ideally be carried out in a vacuum, but here they were performed in air. The movements of the shaker table pumped air through the sample, and the friction between air and fibers influence the measurement. Section II contains a derivation of a new formula used to corrects the data for the influence from airflow through the sample. Section II also shows a new way of correcting for wave movements in the fiber skeleton. The elastic constant can be measure with one sample, in

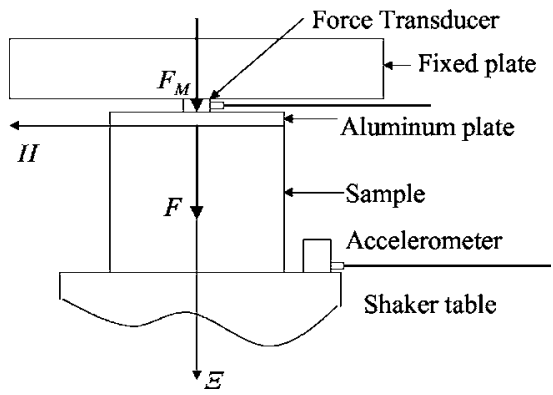

FIG. 2. Measurement principle with one moving surface, the force transducer fixes the aluminum plate. The force on the sample and the acceleration of the shaker table are measured. $\Xi$ and $H$ marks the axes of a coordinate system fixed on the aluminum plate. contrast to the investigation by Wilson and Cummings ${ }^{10}$ that requires two samples and assumed homogeneity of the samples. A new mechanical design is described in Sec. III, which also contains details of the electronic system.

Glass wool is anisotropic, but all the above-cited works present only measurements of one Young's modulus, the smallest one. Dynamic measurements of all the elastic constant are reported in Sec. IV, where all the dynamic elastic constants in the frequency range $20-160 \mathrm{~Hz}$ for glass wool of density $30 \mathrm{~kg} / \mathrm{m}^{3}$ are given. The results are compared with measurements of the elastic properties of glass wool in the papers Refs. 6-10.

\section{PRINCIPLE OF MEASUREMENT}

The elastic properties of glass wool are highly anisotropic. Glass wool mats are fabricated on a conveyer belt on which the glass fibers are laid. The glass wool is soft in the direction perpendicular to the conveyer belt and hard in all directions parallel to the belt. We use a coordinate system with $Z$ axis in the soft direction, $X$ axis in the direction the belt moves, and $Y$ axis perpendicular to the first two axes. The standard nomenclature for elastic constants is used. Call the stress tensor $\sigma_{p q}$ with $p, q=x, y$ or $z$, and define a stress vector $\Sigma=\left(\sigma_{x x}, \sigma_{y y}, \sigma_{z z}, \sigma_{y z}, \sigma_{x z}, \sigma_{x y}\right)$. Call the strain tensor $\varepsilon_{p q}$, and define the strain vector $\mathbf{E}$ $=\left(\varepsilon_{x x}, \varepsilon_{y y}, \varepsilon_{z z}, 2 \varepsilon_{y z}, 2 \varepsilon_{x z}, 2 \varepsilon_{x y}\right)$ : Then the elastic constants matrix $\mathbf{C}$ is defined by $\mathbf{\Sigma}=\mathbf{C E}$.

The $\mathbf{C}$ matrix is symmetrical. Rice and Göransson ${ }^{8}$ state that their light glass wool is transversally isotropic. The glass wool studied her is also assumed transversally isotropic. Due to the symmetry, some components of the elastic constant matrix are zero, and some are equal, which is shown in the following matrix:

$$
\left\{\begin{array}{cccccc}
C_{11} & C_{12} & C_{13} & 0 & 0 & 0 \\
\cdot & C_{11} & C_{13} & 0 & 0 & 0 \\
\cdot & \cdot & C_{33} & 0 & 0 & 0 \\
\cdot & \cdot & \cdot & C_{44} & 0 & 0 \\
\cdot & \cdot & \cdot & \cdot & C_{44} & 0 \\
\cdot & \cdot & \cdot & \cdot & \cdot & \frac{1}{2}\left(C_{11}-C_{12}\right)
\end{array}\right\} .
$$

The form of the matrix is given in Jones. ${ }^{13}$

Rice and Göransson ${ }^{8}$ stated, based on static measurements, that the Poisson's ratios are zero. This is also assumed for the glass wool examined in this paper, and from Jones ${ }^{13}$ it follows that $C_{12}=C_{12}=0$. Thus, the elastic constant matrix is diagonal, and it can be computed from the two Young's moduli $E_{1}, E_{3}$, and the shear module $G$,

$$
\left\{\begin{array}{cccccc}
E_{1} & 0 & 0 & 0 & 0 & 0 \\
\cdot & E_{1} & 0 & 0 & 0 & 0 \\
\cdot & \cdot & E_{3} & 0 & 0 & 0 \\
\cdot & \cdot & \cdot & G & 0 & 0 \\
\cdot & \cdot & \cdot & \cdot & G & 0 \\
\cdot & \cdot & \cdot & \cdot & \cdot & \frac{1}{2} E_{1}
\end{array}\right\} .
$$




\section{A. Calculating Young's modules from data}

All samples were prismatic, cut perpendicular to one of the axes $X, Y$, and $Z$ in the glass wool mats. Figure 2 shows the principle of the setup. The shaker was excited to give its table a simple harmonic motion, and the complex acceleration amplitude of the table $A$ and the frequency $f$ was recorded. The displacement of the table $u_{T}$ can be calculated from the cyclic frequency $\omega=2 \pi f$ and the amplitude of acceleration,

$$
u_{T}=-A / \omega^{2} .
$$

The complex force amplitude $F_{M}$ measured by the force transducer was recorded. The force transducer was mounted on a plate that did not move, fixed plate in Fig. 2, and the force transducer was very stiff. Therefore the aluminum plate, on top of the sample, did not move, and the force on the transducer was equal to the force $F$ on the sample, $F$ $=F_{M}$. Airflow in the sample is neglected, but it will be considered in the next section. The frequency is assumed to be so low that wave motion in the sample may be neglected. This is the case when Young's module $E_{1}$ is determined. Then $E_{1}$ equals the stress divided by the strain, if the $X$ axis of the sample is parallel to the $\Xi$ axis of the apparatus. Stress is the force divided by the cross-section area of the sample $S$, and the strain is the displacement of the table divided by the length of the sample $l$. Thus,

$$
E_{1}=\frac{F / S}{u_{T} / l} .
$$

There is no lateral strain because the elastic constants $C_{12}$ $=0$ and $C_{13}=0$.

\section{B. Airflow in the sample}

During measurements air was pumped in and out of the sample by the movements of the shaker table. The airflow influenced the measurement of the imaginary part of the elastic constant, and this influence is calculated approximately in this section. First the energy loss $P$ due to friction between air and fibers is calculated. An integral of the power loss density over the volume $V$ of the sample gives $P$,

$$
P=\int_{V} R_{r s} v_{r} v_{s} d V .
$$

The velocity $v_{r}$ is referred to the coordinate system in Fig. 2, with axes $\Xi, H$, and $Z$ and coordinates $(\xi, \eta, \varsigma)$. The indices $r, s=\xi, \eta$, or $\mathrm{s}$, and repeated indices imply summation. The airflow resistivity $R_{r s}$ is a tensor because the glass wool is anisotropic. Only the case where the resistivities are equal in all directions perpendicular to the $\Xi$ axis is needed. It is assumed that the air is incompressible, because the wavelength of sound in the air between fibers is much longer than the dimension of the apparatus. The $\Xi$ axis, Fig. 2, is the polar axis of a cylinder coordinate system with coordinates $\xi$, $\rho$, and $\varphi$, where $\rho$ is the distance from the polar axis and $\varphi$ the angle. The radial velocity of air $v_{\rho}$, perpendicular to the $\Xi$ axis is, from the incompressibility of air, approximately

$$
v_{\rho}=\frac{\pi \rho^{2} v_{T}}{2 \pi \rho l}=\frac{\rho v_{T}}{2 l},
$$

where $v_{T}$ is the velocity of the shaker table, Fig. 2, in the $\Xi$ direction. The power $P$ dissipated equals the volume integral over the sample

$$
P=\int_{V} R_{\perp} v_{\rho}^{2} d V,
$$

where $R_{\perp}$ is the airflow resistivity perpendicular to the $\Xi$ axis, and only radial flow is considered. Substitute Eq. (6) into Eq. (7), and get

$$
P=\frac{R_{\perp} v_{T}^{2}}{4 l^{2}} \int_{V} \rho^{2} d V .
$$

If the sample cross-section is square with side length $a$, then the integral becomes

$$
P=\frac{R_{\perp} a^{4} v_{T}^{2}}{24 l} .
$$

The power delivered from the shaker table to the sample is

$$
P=v_{T} F_{V},
$$

where $F_{V}$ is the viscous force. From Eqs. (9) and (10) one gets the force

$$
F_{V}=\frac{R_{\perp} a^{4}}{24 l} v_{T} .
$$

We use a complex time factor $\mathrm{e}^{i \omega t}$, where $t$ is the time and $i^{2}=-1$. Then $v_{T}=i \omega u_{T}$, and Eq. (11) becomes

$$
F_{V}=\frac{R_{\perp} a^{4}}{24 l} i \omega u_{T} .
$$

This force was subtracted from the direct measured force $F_{M}$ to give the force $F$ that would act on the fiber skeleton in vacuum. $F$ is the force that should be used in Eq. (4),

$$
F=F_{M}-F_{V} .
$$

The real part of $F_{M}$ was in principle also influenced by the airflow, but in the present measurement the influence was much smaller that the experimental uncertainty.

\section{Wave motion in the fiber skeleton}

The elastic constant $E_{3}$ is so small that the wavelength of waves in the sample skeleton becomes comparable to the length of the sample, and the strain is not constant in the sample. The calculation of the elastic constant from the data in this case follows in this section.

Figure 2 shows a coordinate system with $\Xi$ axis perpendicular to the aluminum plate and origin in the center of this plate on the sample side. $\xi$ is the coordinate in the $\Xi$ direction, $u$ is the displacement of the fiber skeleton in this direction, $u_{0}$ is a constant, and $k$ is the wave number. The displacement of the sample at the aluminum plate, $\xi=0$, is zero. Therefore, 


$$
u=u_{0} \sin k \xi .
$$

The constant $u_{0}$ can be found from the displacement $u_{T}$ of the shaker table,

$$
u_{0}=u_{T} / \sin k l \text {. }
$$

To find $E_{3}$ the $Z$ axis of the glass wool sample was parallel to the $\Xi$ axis of the apparatus.

The force $F$ on the aluminum plate is

$$
F=\left.S E_{3} \frac{\partial u}{\partial \xi}\right|_{\xi=0} .
$$

Equations (14)-(16) give

$$
\frac{F}{u_{T}}=\frac{S E_{3} k}{\sin k l} .
$$

Equation (17) is solved for $E_{3}$,

$$
E_{3}=\frac{F \sin k l}{u_{T} S k} .
$$

The wave number is the cyclic frequency divided by the wave velocity. If the mass density of the fiber skeleton is $\rho_{W}$, then the wave number is

$$
k=\frac{\omega}{\sqrt{\frac{E_{3}}{\rho_{W}}}} .
$$

In the experiment the acceleration $A$ of the shaker table, Fig. 2 , was measured, and $u_{T}$ was calculated from Eq. (3). The force transducer measured force $F_{M}$, and $F$ was calculated from Eq. (13). [The correction for airflow Eq. (13) is not necessary when $E_{1}$ is calculated because the imaginary part of $E_{1}$ is much higher than the correction by Eq. (13).] $\omega, S$, and $l$ were recorded. The mass density $\rho_{W}$ was assumed to be equal to the static mass density, $30 \mathrm{~kg} / \mathrm{m}^{3}$. All mechanical losses are attributed to the elastic constant. Equations (18) and (19) give implicitly $E_{3}$.

An iterative procedure was used to find $E_{3}$. A complex value of $E_{3}$ was assumed and $k$ was calculated from Eq. (19). This $k$ value was set into Eq. (18) and a new value for $E_{3}$ was computed. Then a new value of $k$ was calculated from Eq. (19). The iteration was stopped when a stationary value of $E_{3}$ was obtained.

\section{MEASUREMENT SETUP}

The mechanical setup used to measure $E_{1}$ and $E_{3}$ is shown in Fig. 3. Four steel rods with threads and nuts held the two, 10-mm-thick, aluminum plates A and D. On plate D was mounted the shaker that carried a 5-mm-thick aluminum plate $\mathrm{C}$, whose acceleration was measured by three piezoelectric accelerometers. They were placed at the vertices of an equilateral triangle with center in the center of the plate $\mathrm{C}$. Three accelerometers were used, instead of one to detect possible rotations of the plate. The glass wool sample was glued to plates B and C. The plate B, 5-mm-thick aluminum, was mounted on plate A via a piezoelectric force transducer. The thickness of the sample was typical $50 \mathrm{~mm}$.
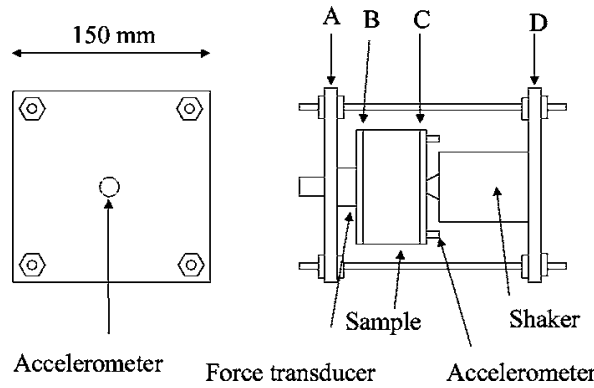

FIG. 3. The mechanical details of the setup used to measure Young's moduli. It uses the measurement principle in Fig. 2. The setup hung in $1 \mathrm{~m}$ long rubber bands. The sample is glued to the two plates B and C.

The whole assembly hung in two 1-m-long rubber bands. These bands are not shown in Fig. 3. In the early experiments, plate $\mathrm{D}$ was mounted horizontally on a concrete slab. But the suspension by rubber bands eliminated many resonance modes that disturbed the measurement and this greatly improved the data. Plate A, the force transducer, and plate B had a small acceleration, which was measured by an accelerometer mounted on plate $\mathrm{A}$. The acceleration and the mass of plate B was used to correct the output from the force transducer to give the actual force from the sample on plate B. The derivation of Eq. (18) assumed that the displacement of plate B was zero. In the actual experiment the displacement was very small compared the movement of plate $\mathrm{C}$, so Eq. (18) can still be used to calculate the influence from wave movement in the skeleton of the sample.

The shear constant $G$ was measured with the setup in Fig. 4. The right picture gives the cut S-S shown on the left-hand side. The sample was glued to plates B and C. The shaker moved plate $\mathrm{C}$ horizontally, and its acceleration was measured by an accelerometer. Plate B was attached to a force transducer, which was fastened on plate A. The force transducer measured the horizontal force on plate $\mathrm{B}$, and the displacement of plate $\mathrm{C}$ was calculated from its measured acceleration. The procedure in Sec. II C was used to correct for wave movements of the fiber skeleton. There was no airflow in the sample because it was subject to a shear deformation.

Figure 5 shows the electronic equipment. It used a Stanford Research System DSP Lock-In Amplifier SR850, which has an output voltage that is a sinusoidal function of time.

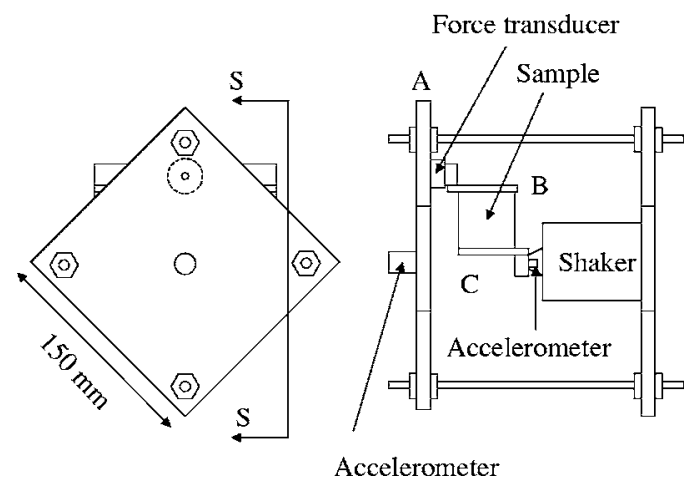

FIG. 4. The mechanical details of the setup used to measure the shear constant. 


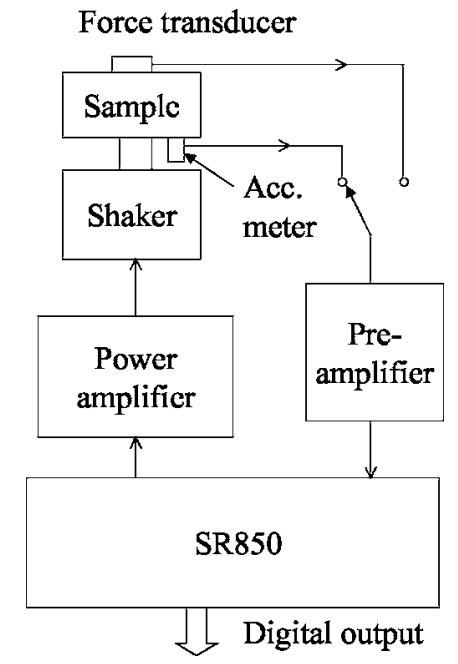

FIG. 5. The electronic setup used to record accelerations and force. The SR850 delivers a voltage that is a sinusoidal function of time. This voltage drove the power amplifier, which was connected to the shaker. The accelerometer or the force transducer was connected to the preamplifier. The amplitude and phase of the output from the preamplifier was measured and recorded by SR850.

The frequency can be programmed to sweep through a range. The output voltage from SR850 was connected to a power amplifier that drove the shaker. SR850 also has an input to a phase detector, and SR850 can record digitally the magnitude and phase of the input. One accelerometer on plate $\mathrm{C}$ was connected to a preamplifier, the output of which was connected to the input of SR850. The frequency was swept through a range, and the frequency, the magnitude, and phase of the signal from the accelerometer were recorded digitally. Then a new accelerometer was connected to the preamplifier and the procedure repeated. After all the accelerometer outputs were recorded, the force transducer was connected to the preamplifier, and the magnitude and phase of the force were recorded.

\section{RESULTS}

The glass wool studied was of the type "Industriplade 1" produced by Saint-Gobain Isover a/s, Vamdrup, Denmark. Table I shows data for the glass wool.

The $Z$ resistivity was measured with airflow in the $Z$ direction in the glass wool, defined in Sec. II, and $X$ resistivity was measured with airflow in the $X$ direction. Resistivity in the $X$ and $Y$ directions are equal. The porosity was calculated from $1-\rho_{W} / \rho_{F}$, where $\rho_{W}$ is the density of glass wool, and $\rho_{F}$ is the density of fiber glass.

TABLE I. Acoustic data for "Industriplade 1."

\begin{tabular}{lr}
\hline \hline Mass density of glass wool & $30 \mathrm{~kg} / \mathrm{m}^{3}$ \\
Mass density of fiber glass & $2550 \mathrm{~kg} / \mathrm{m}^{3}$ \\
Diameter of fiber & $7 \mu \mathrm{m}$ \\
Airflow Z-resistivity & $16 \mathrm{kPa} \mathrm{s} \mathrm{m}^{-2}$ \\
Airflow X-resistivity & $8 \mathrm{kPa} \mathrm{s} \mathrm{m}^{-2}$ \\
Concentration of phenol-formaldehyde resin & $5 \%$ \\
Porosity & 0.988 \\
\hline \hline
\end{tabular}

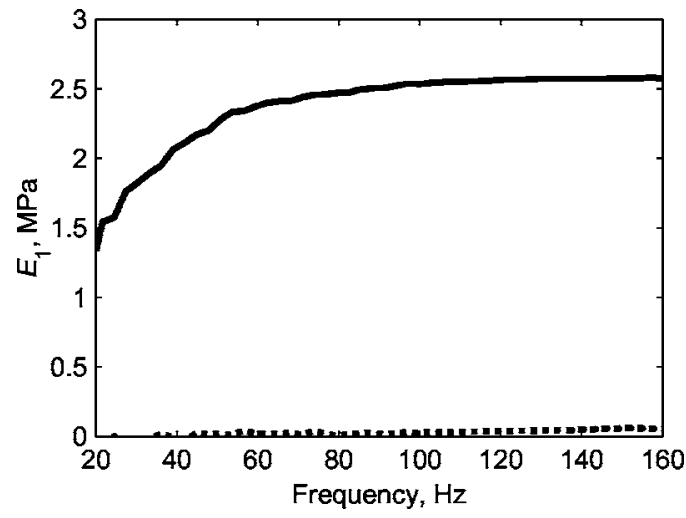

FIG. 6. The resulting Young's module $E_{1}$. The solid line shows the real part and the dotted one the imaginary part. Both real and imaginary part increases with frequency. At $20 \mathrm{~Hz} E_{1}=1.5+0.01 i \mathrm{MPa}$, and at $160 \mathrm{~Hz} E_{1}$ $=2.6+0.06 i \mathrm{MPa}$.

\section{A. Young module $E_{1}$}

The sample had a cross-section area of $100 \cdot 100 \mathrm{~mm}^{2}$ perpendicular to the $X$ axis in the glass wool. Its length was $50 \mathrm{~mm}$. The sample was glued to the two plates B and C in Fig. 3. Figure 6 shows the resulting real and imaginary part of $E_{1}$. The solid curve is the real part and the dotted one the imaginary part. It was necessary to use a low level of acceleration to avoid elastic nonlinearity in the glass wool sample. The strain used for Fig. 6 was below $10^{-5}$.

There are few data in the literature that the result can be compared with, and only static measurement seems to have been published. Rice and Göransson ${ }^{8}$ reported a Young modulus $E_{1}=17.2 \mathrm{kPa}$ for glass wool of mass density $14 \mathrm{~kg} / \mathrm{m}^{3}$. Tarnow ${ }^{14}$ found the static value $390 \mathrm{kPa}$ for glass wool of mass density $30 \mathrm{~kg} / \mathrm{m}^{3}$, and the dynamic result for the real part was $1.5 \mathrm{MPa}$ at $20 \mathrm{~Hz}$. The strain was $2 \cdot 10^{-2}$ by the static measurement, and below $10^{-5}$ by the dynamic one. The static strain is much higher than the dynamic one, and nonlinear elasticity could explain the difference between the static and the dynamic value.

\section{B. Young's modulus $E_{3}$}

The sample had a cross-section area of $100 \cdot 100 \mathrm{~mm}^{2}$ perpendicular to the $Z$ axis in the glass wool. Its length was $31 \mathrm{~mm}$. Figure 7 shows the result for $E_{3}$ for the sample of glass wool. The data show that the real and imaginary parts are constants. The best graphical fit of the data was $E_{3}=12$ $+0.6 i \mathrm{kPa}=12(1+0.05 i) \mathrm{kPa}$.

The real part of $E_{3}$ is more than 100 times smaller than the one of $E_{1}$. The strain used for Fig. 7 was below $10^{-4}$ to avoid elastic nonlinearity. A higher strain would have reduced to the electronic noise.

Equtions (12) and (13) with an airflow resistivity $=8 \mathrm{kPa} \mathrm{s} \mathrm{m}^{-2}$ were used to correct for the influence from airflow in the sample. Wave movements in the fiber skeleton were taken into account by Eqs. (18) and (19).

Wilson and Cummings ${ }^{10}$ reported dynamic measurements for glass wool of density $24 \mathrm{~kg} / \mathrm{m}^{3}$. They found an approximately constant $E_{3}=3.3+0.2 i \mathrm{kPa}=3.3(1+0.06 i)$ $\mathrm{kPa}$ in the frequency range $40-200 \mathrm{~Hz}$. The real part is four times smaller than the one found here, but the imaginary

Viggo Tarnow: Dynamic measurements of elastic constants 


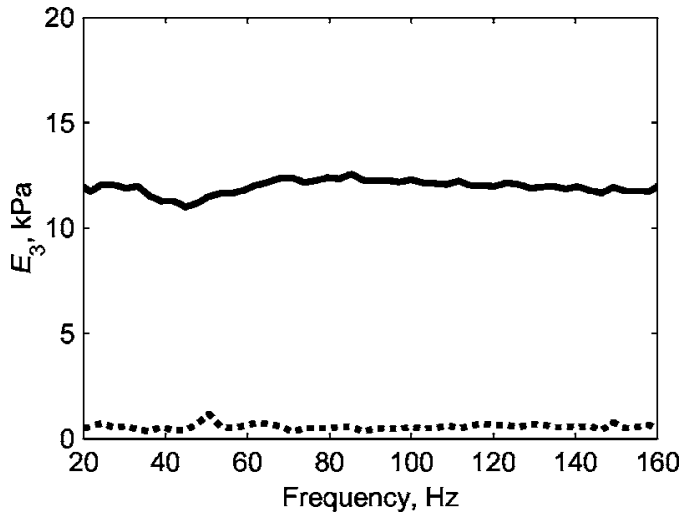

FIG. 7. The resulting Young's module $E_{3}=12+0.6 i \mathrm{kPa}$. The solid line shows the real part and the dotted one the imaginary part. Both the real and imaginary parts are constant.

parts divided by the real ones, the loss factors, are approximately equal. For the glass wool Telwolle of density $71 \mathrm{~kg} / \mathrm{m}^{3}$ and fiber diameter 5-7 $\mu \mathrm{m}$, Pritz $^{7}$ reported a constant complex Young's modulus $E_{3}=78.4(1+0.02 \cdot i) \mathrm{kPa}$ in the frequency range $100-3000 \mathrm{~Hz}$.

\section{Shear modulus $G$}

The sample had dimensions $100 \cdot 100 \cdot 31 \mathrm{~mm}^{3}$ in the $X$, $Y, Z$ direction of the glass wool. The setup in Fig. 4 was used with $31 \mathrm{~mm}$ between plates $\mathrm{B}$ and $\mathrm{C}$. The direction of displacement was along the $100 \mathrm{~mm}$ edges in the $X$ direction. The result is shown in Fig. 8. The data are only reliable for frequencies below $100 \mathrm{~Hz}$, because of resonance in the mechanical structure. In the frequency range $20-100 \mathrm{~Hz}$ the shear modulus is constant and equal to $40+2 i \mathrm{kPa}$.

Only static measurements of this module have been published. Rice and Göransson ${ }^{7}$ reported $13.7 \mathrm{kPa}$ for glass wool of density $14 \mathrm{~kg} / \mathrm{m}^{3}$.

\section{Poisson's ratios}

Rice and Göransson ${ }^{8}$ stated, based on static measurements, that the Poisson's ratios are zero for glass wool of mass density 15 and $11 \mathrm{~kg} / \mathrm{m}^{3}$. This means that $C_{12}=C_{13}$ $=0$ from Jones. ${ }^{13}$

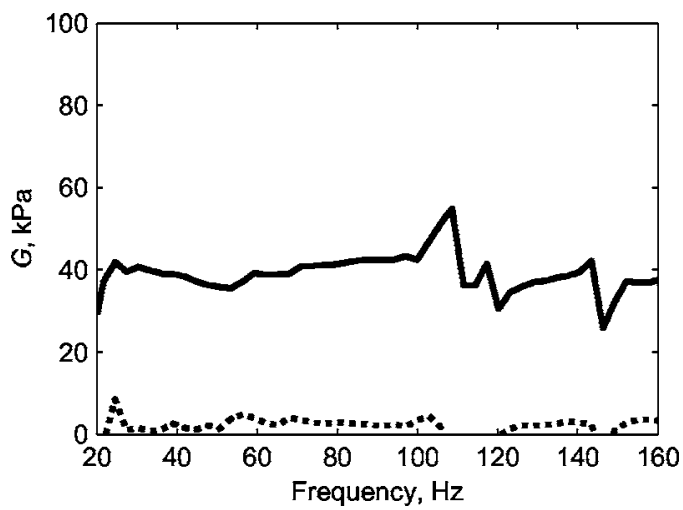

FIG. 8. The resulting shear module $G=40+2 i \mathrm{kPa}$. The solid line shows the real part and the dotted one the imaginary part. Both the real and imaginary part are constant. For frequencies above $100 \mathrm{~Hz}$ the result is not accurate due to mechanical resonance.
It was attempted to measure the Poisson's ratios dynamically. A sample of glass wool was placed between two parallel aluminum plates. An electrodynamic shaker moved one of the plates. To detect the movement of the sides of the sample, aluminum foils $20 \mu \mathrm{m}$ thick were glued on the sides of the sample, and a preamplifier for a condenser microphone was used to detect lateral movements of the sample. However, the scattering of the data made it impossible to detect movements of the sides of the sample.

Another attempt was made to measure Poisson's ratios. The apparent Young's modulus was found for samples of different lengths by the method in Sec. II. When the sample length is small compared to the dimensions of the aluminum plates, the deformation of the sample is perpendicular to the aluminum plates, because they prevent lateral movement. In this case one would find a high value of the Young's modulus. If the sample is long, there is lateral movement depending on the size of Poisson's constants, and this lateral movement would decrease the apparent Young's module. No such dependence on length was found, but the data were scattered due to inhomogeneity of the samples. Therefore the Poisson's ratios are assumed to be zero.

\section{CONCLUSION}

A new mechanical design for measuring the elastic constants of glass has been developed. Correction for viscous drag on fibers from airflow in the sample is presented, and correction for mechanical waves in the structure is described. The measurement showed that the Young's module $E_{1}$ depended on frequency, at $20 \mathrm{~Hz}$ it was $1.5+0.01 \mathrm{MPa}$, and at $160 \mathrm{~Hz}$ it was $2.6+0.06 i \mathrm{MPa}$. Young's module $E_{3}=12$ $+0.6 i \mathrm{kPa}$ did not depend on frequency. The shear module $G=40+2 i \mathrm{kPa}$ did not depend on frequency. Attempts to measure the Poisson's ratios did not give conclusive results, and they were assumed to be zero.

\section{ACKNOWLEDGMENT}

Myhrwold's fund, Denmark, supported the work reported here.

${ }^{1}$ T. E. Vigran, L. Kelders, W. Lauriks, P. Leclaire, and T. F. Johansen, "Prediction and measurements of the influence of boundary conditions in a standing wave tube," Acust. Acta Acust. 83, 419-423 (1997).

${ }^{2}$ B. H. Song and J. S. Bolton, "Effect of circumferential edge constraint on the acoustic properties of glass fiber materials," J. Acoust. Soc. Am. 110, 1902-2916 (2001).

${ }^{3}$ B. H. Song and J. S. Bolton, "Investigation of the vibrational modes of edge-constrained fibrous samples placed in standing wave tube," J. Acoust. Soc. Am. 113, 1833-1849 (2003).

${ }^{4} \mathrm{~V}$. Tarnow, "Fiber movements and sound attenuation in glass wool," J. Acoust. Soc. Am. 105, 234-240 (1999).

${ }^{5}$ M. A. Biot, "Theory of Propagation of Elastic Waves in a Fluid-Saturated Porous Solid. I. Low-Frequency Range," J. Acoust. Soc. Am. 28, 168-178 (1956).

${ }^{6} \mathrm{~T}$. Pritz, "Transfer function method for investigating the complex modulus of acoustic materials: spring-like specimen," J. Sound Vib. 72, 317-341 (1980).

${ }^{7}$ T. Pritz, "Frequency dependence of the frame dynamic characteristics of mineral and glass wool materials," J. Sound Vib. 106, 161-169 (1986).

${ }^{8}$ H. J. Rice and P. Göransson, "A dynamical model of light fibrous materials," Int. J. Mech. Sci. 41, 561-579 (1999).

${ }^{9}$ K. U. Ingard, Notes on Sound Absorption Technology (Noise Control Foundation, Poughkeepsie, New York, 1994). 
${ }^{10} \mathrm{R}$. Wilson and A. Cummings, "An impedance technique for determining the dynamic structural properties of a porous material," Proceedings of the 1996 International Congress on Noise Control Engineering, Liverpool, UK, pp. 269-272.

${ }^{11}$ C. Langlois, R. Panneton, and N. Atalla, "Polynominal relations for quasistatic mechanical characterization of isotropic poroelatic materials," J. Acoust. Soc. Am. 110, 3032-3040 (2001).
${ }^{12}$ L. Cremer, M. Heckl, and E. E. Ungard, Structure-Borne Sound (Springer, Berlin, 1973), p. 198.

${ }^{13}$ R. M. Jones, Mechanics of Composite Materials (Hemisphere, New York, 1975), pp. 32-33, 35.

${ }^{14} \mathrm{~V}$. Tarnow, "Measurements of anisotropic sound propagation in of glass wool,” J. Acoust. Soc. Am. 101, 2243-2247 (2000). 